\title{
Anaemia and Related Nutritional Deficiencies in Chinese Patients with Obesity, 12 Months Following Laparoscopic Sleeve Gastrectomy
}

This article was published in the following Dove Press journal: Diabetes, Metabolic Syndrome and Obesity: Targets and Therapy

\author{
Chunlan Zhang' \\ Xi Chen' \\ Jingping $\mathrm{Li}^{2}$ \\ Zhenqi Liu $^{3}$ \\ Wei Liu ${ }^{2}$ \\ Jingjing Zhang (ID \\ Zhiguang Zhou'
}

'National Clinical Research Center for Metabolic Diseases, Metabolic Syndrome Research Center, Key Laboratory of Diabetes Immunology, Ministry of Education, Department of Metabolism and Endocrinology, The Second Xiangya Hospital of Central South University, Changsha, Hunan, 4I00 I I, People's Republic of China; ${ }^{2}$ Department of Metabolic Surgery, Department of Biliopancreatic Surgery, The Second Xiangya Hospital, Central South University, Changsha, Hunan, 4I00II, People's Republic of China; ${ }^{3}$ Division of Endocrinology and Metabolism, Department of Medicine, University of Virginia Health System, Charlottesville, VA, USA

Correspondence: Jingjing Zhang National Clinical Research Center for

Metabolic Diseases, Metabolic Syndrome

Research Center, Key Laboratory of

Diabetes Immunology, Ministry of

Education, Department of Metabolism and Endocrinology, The Second Xiangya Hospital of Central South University, I39

Renmin Middle Road, Changsha, Hunan,

People's Republic of China

Tel +86 73I-85292154

Email doctorzhangij@csu.edu.cn

Wei Liu

Department of Metabolic Surgery,

Department of Biliopancreatic Surgery,

The Second Xiangya Hospital, Central

South University, 139 Renmin Middle

Road, Changsha, Hunan, People's Republic of China

Tel $+86731-85292154$

Email liuweixy@csu.edu.cn
Background: Laparoscopic sleeve gastrectomy (LSG) has become a predominant bariatric procedure at present. However, data are scarce regarding the nutritional impact of this procedure on Chinese patients. This study aimed to evaluate the prevalence of nutritional deficiency after LSG in Chinese patients.

Methods: Eighty-two patients with obesity were recruited from the Second Xiangya Hospital of Central South University, and all patients underwent LSG and completed the visit.

Results: Compared with the baseline, the serum albumin levels increased significantly at 1-12 months $(P<0.001)$ after surgery, and the hypoalbuminemia rate decreased from $8.5 \%$ to $0 \%$ throughout the study $(P=0.063)$. Anaemia was present in $7.3 \%$ of all patients before surgery, and its prevalence increased to $11.0 \%$ at 12 months post-operation $(P=0.109)$. The anaemia rate of fertile females was higher than that of males $(21.4 \%$ vs $2.3 \%, P=0.036)$. No significant changes were found in vitamin B12 deficiency throughout the study $(0 \%$ vs $3.8 \%$, $P=1.0$ ). The increases in the folate deficiency were only discovered in the female group (3.7\% vs $20 \%, P=0.031)$ and the obese without type 2 diabetes (T2D) group after LSG (27.3\% vs $47.1 \%, P=0.031)$. A decrease in the ferritin levels and an increase in iron deficiency at 12 months post-surgery were found among all patients.

Conclusion: Based on 12 months of follow-up, LSG is effective in controlling metabolic syndrome and has a modest effect on nutritional deficiencies, which suggests that LSG is an effective and comparably safe procedure for Chinese patients considering nutritional deficiencies at 12 months post-surgery.

Keywords: anaemia, laparoscopic sleeve gastrectomy, nutrition, obesity

\section{Introduction}

Obesity is highly prevalent worldwide and is a risk factor for type 2 diabetes (T2D), cardiovascular disease and even some cancers. ${ }^{1-4}$ Approximately 641 million people are with obesity worldwide; notably, according to the current study, the obese population in China accounts for $14.0 \%$ of the total obese population in the world. ${ }^{2}$ Metabolic surgery has become a major therapeutic option to treat obesity and its related diseases due to the long-term positive effect on sustained weight loss and overall improved metabolic outcomes. ${ }^{5-7}$

Four kinds of metabolic surgery including laparoscopic sleeve gastrectomy (LSG), laparoscopic Roux-en-Y gastric bypass (LRYGB), laparoscopic adjustable gastric banding and laparoscopic biliopancreatic diversion are well-used surgical 
options. Although LSG and LRYGB are similarly effective in terms of weight loss and glycaemic control, LSG is much easier to perform than LRYGB. ${ }^{7}$ Laparoscopic adjustable gastric banding has gradually fallen out of favour due to a high rate of requiring a subsequent surgery. ${ }^{8}$ Laparoscopic biliopancreatic diversion engenders much higher rates of post-surgical, nutrition-related complications than other bariatric surgeries. ${ }^{9,10}$ Thus, currently, the most common used procedure is LSG worldwide.

After years of practice and short-term to long-term follow-up, studies have shed light on the effect of bariatric surgery on the nutritional status of subjects with obesity. There was evidence demonstrating significantly lower rates of anaemia ( $16.7 \%$ vs $42.9 \%)$ preoperatively compared to 12-20 months postoperatively in Israel after LRYGB. ${ }^{11}$ Decreased albumin and ferritin levels five years after LSG were found in a Canadian study. ${ }^{12}$ Iron deficiency and iron deficiency anaemia were extremely frequent in Chinese patients with obesity and T2D after LRYGB. ${ }^{13}$ To date, little is known about the nutritional status of Chinese patients after LSG. This study focused on the impact of LSG on the nutritional deficiencies of Chinese subjects.

\section{Materials and Methods}

\section{Participants}

In this observational cohort study, eighty-two patients with obesity ( 44 males and 38 females) were recruited from the Second Xiangya Hospital of Central South University (Changsha, Hunan, China). This study protocol was conducted in accordance with the Declaration of Helsinki and was approved by the ethics committee of the Second Xiangya Hospital of CSU, and informed consent of the study protocol was provided by all participants. All subjects accepting LSG met the criteria for metabolic surgery, ${ }^{14}$ including 52 subjects $(63.4 \%)$ had T2D, 30 subjects $(36.5 \%)$ normal glucose tolerance, and 54 subjects $(65.8 \%)$ dyslipidemia. T2D was diagnosed according to World Health Organization criteria. ${ }^{15}$ The subjects with normal glucose tolerance underwent a standardized 75 -g oral glucose tolerance test for normoglycemia (fasting plasma glucose $[\mathrm{FPG}]<6.1 \mathrm{mmol} / \mathrm{L}, 2$-h plasma glucose $<7.8 \mathrm{mmol} / \mathrm{L}$ ). The exclusion criteria were the regular use of anti-inflammatory drugs or corticosteroid medications; type 1 diabetes; secondary diabetes; inflammation, infectious diseases, or other autoimmune disorders; pregnancy; and malignant diseases.
All participants were instructed to take one multivitamin complex tablet a day, containing vitamin B12 $4 \mu \mathrm{g}$, folate $400 \mu \mathrm{g}$, iron $18 \mathrm{mg}$, vitamin B2 $1.7 \mathrm{mg}$, vitamin B6 $2 \mathrm{mg}$, vitamin C $60 \mathrm{mg}$, vitamin E $30 \mathrm{mg}$ and vitamin D 400 IU.

\section{Study Design and Laboratory Analysis}

Study physicians recorded anthropometric data including the body height and weight, waist circumference, hip circumference, and blood pressure before and 1 month, 2 months, 6 months, and 12 months after the therapy. Fasting venous blood samples were tested for FPG, haemoglobin A1C (HbA1c), fasting C-peptide (FCP), total cholesterol (TC), low-density lipoprotein cholesterol (LDL-C), high-density lipoprotein cholesterol (HDL-C), triglycerides (TGs), complete blood count, albumin, vitamin B12, folate, ferritin, and iron. Twohour venous blood samples were tested for post-prandial plasma glucose (PPG) and post-prandial C-peptide (PCP). HbAlc was measured by automated liquid chromatography (VARIANT II Haemoglobin Testing System; Bio-Rad Laboratories, Hercules, CA). C-peptide (CP) was measured by a chemiluminescence method (ADVIA Centaur; Siemens, Munich, Germany). The percentage of excess body mass index (BMI) loss was defined as $(100 \times$ [baseline BMI - follow-up BMI]/ [baseline BMI - 25]).

\section{Definitions of Anaemia and Nutrition Deficiencies}

Hypoalbuminemia was defined by serum levels $<35 \mathrm{~g} / \mathrm{L}$. Anaemia was defined as a haemoglobin $(\mathrm{Hb})$ level $<130 \mathrm{~g} / \mathrm{L}$ in men and $<120 \mathrm{~g} / \mathrm{L}$ in women, and the anaemia severity was classified as mild $(\mathrm{Hb}>110 \mathrm{~g} / \mathrm{L})$, moderate $(\mathrm{Hb}$ between 80 and $110 \mathrm{~g} / \mathrm{L})$, and severe $(\mathrm{Hb}<80 \mathrm{~g} / \mathrm{L}){ }^{16}$ The vitamin B12 deficiency and folate deficiency were defined by serum levels $<203 \mathrm{pg} / \mathrm{mL}$ and $<4 \mathrm{ng} / \mathrm{mL}$, respectively. ${ }^{17}$ The iron deficiency was defined as a ferritin level $<30 \mathrm{ng} / \mathrm{mL} .{ }^{18}$ Microcytic hypochromic anaemia was defined by a mean corpuscular volume $(\mathrm{MCV})<80 \mathrm{fl}$, a mean corpuscular haemoglobin $(\mathrm{MCH})$ $<26 \mathrm{~g} / \mathrm{dL}$, and a mean corpuscular haemoglobin concentration $(\mathrm{MCHC})<32 \%$. Normocytic anaemia was defined as $80 \leq \mathrm{MCV} \leq 100 \mathrm{fl}, 26 \leq \mathrm{MCH} \leq 32 \mathrm{~g} / \mathrm{dL}$, and $32 \%$ $\leq \mathrm{MCHC} \leq 35 \%$. Macrocytic anaemia was defined as $\mathrm{MCV}>100 \mathrm{fl}, \mathrm{MCH}>32 \mathrm{~g} / \mathrm{dL}$, and $\mathrm{MCHC}>35 \%$. 


\section{Surgical Procedure}

All LSG surgeries were performed at the Department of Metabolic Surgery in Xiangya Second Hospital of Central South University.

\section{Statistical Analysis}

The data are presented as the mean \pm the standard deviation (SD) for normally distributed parameters or as the median (25th-75th percentile) for nonnormally distributed parameters. Logarithmic transformations were performed for nonnormally distributed parameters. To compare changes from the baseline to the post-treatment time points within groups, repeated measures of the analysis of variance (ANOVA) with Bonferroni post hoc testing was applied in patients with a complete data set. For binary variable-related samples, McNemar tests were used to analyse the differences among the coupled time points. Subgroup comparisons (between genders or T2D) in the prevalence of deficiencies were performed by Chi-square tests or the Fisher exact test. For the statistical analysis, we used SPSS version 23.0 (IBM Corporation, Chicago, IL) software. A two-tailed $\mathrm{P}<0.05$ was considered statistically significant.

\section{Results}

\section{LSG is Effective for Patients with Obesity in Weight Loss and Metabolic Control}

The baseline and follow-up data of all participants after LSG are presented in Table 1. A total of 82 patients (mean age $31.9 \pm 10.7$ years and mean BMI $40.3 \pm 6.9 \mathrm{~kg} / \mathrm{m}^{2}$ ) underwent LSG. Forty-four participants were men, and 38 were women. The mean BMI of all patients decreased significantly from $40.3 \pm 6.9 \mathrm{~kg} / \mathrm{m}^{2}$ at baseline to $27.1 \pm 3.4 \mathrm{~kg} / \mathrm{m}^{2}$ at 12 months postoperatively. The estimated mean percentage of excess BMI loss at 12 months was $90.2 \%$. Overall, the FBG $(6.8 \pm 3.0 \mathrm{mmol} / \mathrm{L}$ vs $4.7 \pm 0.9 \mathrm{mmol} / \mathrm{L}, P<0.001)$ and TG $(2.4 \pm 1.4 \mathrm{mmol} / \mathrm{L}$ vs $1.2 \pm 1.0 \mathrm{mmol} / \mathrm{L}, P<0.001)$ levels

Table I Anthropometric and Laboratory Data of All Subjects at Baseline and I Month, 2 Months, 6 Months and I2 Months After LSG

\begin{tabular}{|c|c|c|c|c|c|}
\hline & \multicolumn{5}{|c|}{ LSG $(n=82)$} \\
\hline & Baseline & I Month & 2 Months & 6 Months & 12 Months \\
\hline Age (years) & $31.9 \pm 10.7$ & - & - & - & - \\
\hline Sex (female/male) & $(38 / 44)$ & - & - & - & - \\
\hline $\operatorname{BMI}\left(\mathrm{Kg} / \mathrm{m}^{2}\right)$ & $40.3 \pm 6.9$ & $35.1 \pm 7.0 * * *$ & $33.2 \pm 4.9 * * *$ & $29.3 \pm 3.9 * * *$ & $27.1 \pm 3.4^{* * *}$ \\
\hline Excess BMI Loss (\%) & - & $38.2 \pm 30.3$ & $51.2 \pm 22.1$ & $76.4 \pm 25.6$ & $90.2 \pm 25.9$ \\
\hline Waist Circumference (cm) & $122.8 \pm 14.4$ & $114.1 \pm 12.6^{* * *}$ & $108.1 \pm 12.4^{* * *}$ & $97.8 \pm 10.4^{* * *}$ & $91.8 \pm 14.2 * * *$ \\
\hline Hip Circumference $(\mathrm{cm})$ & $122.6 \pm 13.7$ & $116.2 \pm 11.7^{* * *}$ & || $2.7 \pm|| .4 * * *$ & $105.2 \pm 8.8 * * *$ & $101.0 \pm 7.2 * * *$ \\
\hline Waist to Hip Ratio & $1.0 \pm 0.1$ & $0.98 \pm 0.1$ & $0.96 \pm 0.1 * * *$ & $0.92 \pm 0.1 * * *$ & $0.9 \pm 0.1^{* * *}$ \\
\hline $\mathrm{SBP}(\mathrm{mmHg})$ & $137.2 \pm 22.5$ & $122.7 \pm 16.7^{* *}$ & $|20.7 \pm| 4.8^{* *}$ & $121.3 \pm 18.1^{* *}$ & $117.0 \pm 21.5 * *$ \\
\hline $\mathrm{DBP}(\mathrm{mmHg})$ & $84.7 \pm 15.6$ & $82.4 \pm 10.8$ & $82.5 \pm 10.3$ & $78.4 \pm \mid 3.1^{*}$ & $76.4 \pm 12.0^{*}$ \\
\hline TG (mmol/L) & $2.4 \pm 1.4$ & $1.6 \pm 0.5^{* * * *}$ & $1.6 \pm 0.7 * * *$ & $1.2 \pm 0.6 * * *$ & $1.2 \pm 1.0 * * *$ \\
\hline $\mathrm{TC}(\mathrm{mmol} / \mathrm{L})$ & $4.4 \pm 0.9$ & $4.4 \pm 0.9$ & $4.6 \pm 1.0$ & $4.8 \pm 1.0 *$ & $4.7 \pm 1.0 *$ \\
\hline $\mathrm{HDL}(\mathrm{mmol} / \mathrm{L})$ & $1.0 \pm 0.2$ & $1.2 \pm 0.7$ & $1.1 \pm 04$ & $1.2 \pm 0.2^{* * *}$ & $1.4 \pm 0.3 * * *$ \\
\hline $\mathrm{LDL}(\mathrm{mmol} / \mathrm{L})$ & $2.8 \pm 0.8$ & $2.6 \pm 1.0$ & $3.1 \pm 0.8$ & $3.1 \pm 0.9$ & $3.0 \pm 1.0$ \\
\hline FPG (mmol/L) & $6.8 \pm 3.0$ & $5.2 \pm 1.2 * * *$ & $4.9 \pm 0.9 * * *$ & $4.6 \pm 1.1 * * *$ & $4.7 \pm 0.9 * * *$ \\
\hline PPG (mmol/L) & $9.9 \pm 4.6$ & $5.7 \pm 1.9 * * *$ & $5.5 \pm 1.5^{* * *}$ & $5.3 \pm 2.1^{* * *}$ & $5.0 \pm 1.2^{* * *}$ \\
\hline HbAlc (\%) & $7.3 \pm 2.0$ & $6.1 \pm 1 . I^{* * *}$ & $5.4 \pm 0.6 * * *$ & $5.3 \pm 0.5^{* * *}$ & $5.3 \pm 0.5^{* * *}$ \\
\hline FCP (pmol/l/) & II $29.4 \pm 58 \mid .9$ & $913 \pm 480.0$ & $789.3 \pm 330.9 * * *$ & $622.9 \pm 266.9 * * *$ & $518.1 \pm 207.4^{* * *}$ \\
\hline PCP (pmol/L) & $2446.7 \pm 1067.5$ & $1167.0 \pm 70 \mid .6$ & $1113.7 \pm 656.7$ & $1027.2 \pm 621.0$ & $905.8 \pm 487.1$ \\
\hline Albumin $(\mathrm{g} / \mathrm{L})$ & $39.5 \pm 3.5$ & $43.3 \pm 3.5^{* * *}$ & $43.5 \pm 3.5^{* * *}$ & $43.5 \pm 3.5^{* * *}$ & $44.1 \pm 3.1 * * *$ \\
\hline $\mathrm{Hb}(\mathrm{g} / \mathrm{L})$ & $143.6 \pm 18.5$ & $140.0 \pm 16 . \mid$ & $140.2 \pm 16.3$ & $140.1 \pm 17.9$ & $140.7 \pm 16.6$ \\
\hline Vitamin $\mathrm{B} / 2(\mathrm{pg} / \mathrm{mL})$ & $431.7 \pm 118.7$ & $670.9 \pm 313.8^{* * *}$ & $477.8 \pm 193.3^{\# \# \#}$ & $374.7 \pm 217.8^{\# \# \$}$ & $373.0 \pm 171.1^{\# \# \# \$ ~}$ \\
\hline Folate $(\mathrm{ng} / \mathrm{mL})$ & $6.0 \pm 3.1$ & $6.5 \pm 3.0$ & $5.7 \pm 2.6$ & $6.7 \pm 3.9$ & $7.1 \pm 4.6$ \\
\hline Iron (umol/L) & $19.5 \pm 8.0$ & $16.3 \pm 6.3$ & $19.0 \pm 9.8$ & $20.3 \pm 7.1^{\# \#}$ & $22.1 \pm 8.5^{\# \# \#}$ \\
\hline Ferritin(ng/mL) & $247.4 \pm 213.3$ & $361.7 \pm 296.2 *$ & $261.7 \pm 215.5^{\# \# \# ~}$ & 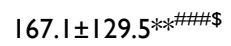 & $167.8 \pm 167.2 * * * \ldots$ \\
\hline
\end{tabular}

Notes: Data are presented as the mean \pm SD. ${ }^{*} p<0.05,{ }^{* *} p<0.01,{ }^{* * *} p<0.001$ compared with baseline; ${ }^{\#} p<0.01,{ }^{\# \#} p<0.001$ compared with I month; ${ }^{\$} p<0.05,{ }^{\$} p<0.01,{ }^{\$}$ $\$ p<0.00$ I compared with 2 months.

Abbreviations: LSG, laparoscopic sleeve gastrectomy; BMI, body mass index; SBP, systolic blood pressure; DBP, diastolic blood pressure; TGs, triglycerides; TC, total cholesterol; HDL, high-density lipoprotein cholesterol; LDL, low-density lipoprotein cholesterol; FPG, fasting plasma glucose; PPG, post-prandial plasma glucose; HbAIc, haemoglobin AIC; FCP, fasting C-peptide; PCP, post-prandial C-peptide; Hb, haemoglobin. 
decreased, and the HDL $(1.0 \pm 0.2 \mathrm{mmol} / \mathrm{L}$ vs 1.4 $\pm 0.3 \mathrm{mmol} / \mathrm{L}, P<0.001)$ levels increased substantially after surgery, while there was a slight increase in TC levels (4.4 $\pm 0.9 \mathrm{mmol} / \mathrm{L}$ vs $4.7 \pm 1.0 \mathrm{mmol} / \mathrm{L}, P=0.013)$. Improvements were also observed in the blood pressure (137.2 \pm 22.5 $\mathrm{mmHg}$ vs $117.0 \pm 21.5 \mathrm{mmHg}, P=0.003 ; 84.7 \pm 15.6 \mathrm{mmHg}$ vs $76.4 \pm 12.0 \mathrm{mmHg}, P=0.024)$.

\section{Serum Albumin Levels Increased Significantly After Surgery}

As shown in Tables 1-3 and Figure 1A, C and E, the serum albumin levels increased significantly at 1 month $(P<0.001), 2$ months $(P<0.001), 6$ months $(P<0.001)$ and 12 months $(P<0.001)$ after surgery. The prevalence of hypoalbuminemia decreased from $8.5 \%$ to $0 \%$ throughout the whole study, although the decrease was not statistically significant $(P=0.063)$ (Figure 1B). No significant differences were found in the hypoalbuminemia rate of each subgroup at the 12month follow-up (Figure 1D and F). The mean levels of albumin in the male group and the female group were similar at baseline $(39.9 \pm 3.8 \mathrm{~g} / \mathrm{L}$ vs $39.1 \pm 3.0 \mathrm{~g} / \mathrm{L}, P>0.05)$, and both increased significantly at the 12 -month visit $(44.8 \pm 3.2 \mathrm{~g} / \mathrm{L}$, $P<0.001 ; 43.1 \pm 2.7 \mathrm{~g} / \mathrm{L}, P<0.001)$. However, the men had significantly higher albumin levels $(44.8 \pm 3.2 \mathrm{~g} / \mathrm{L})$ than the
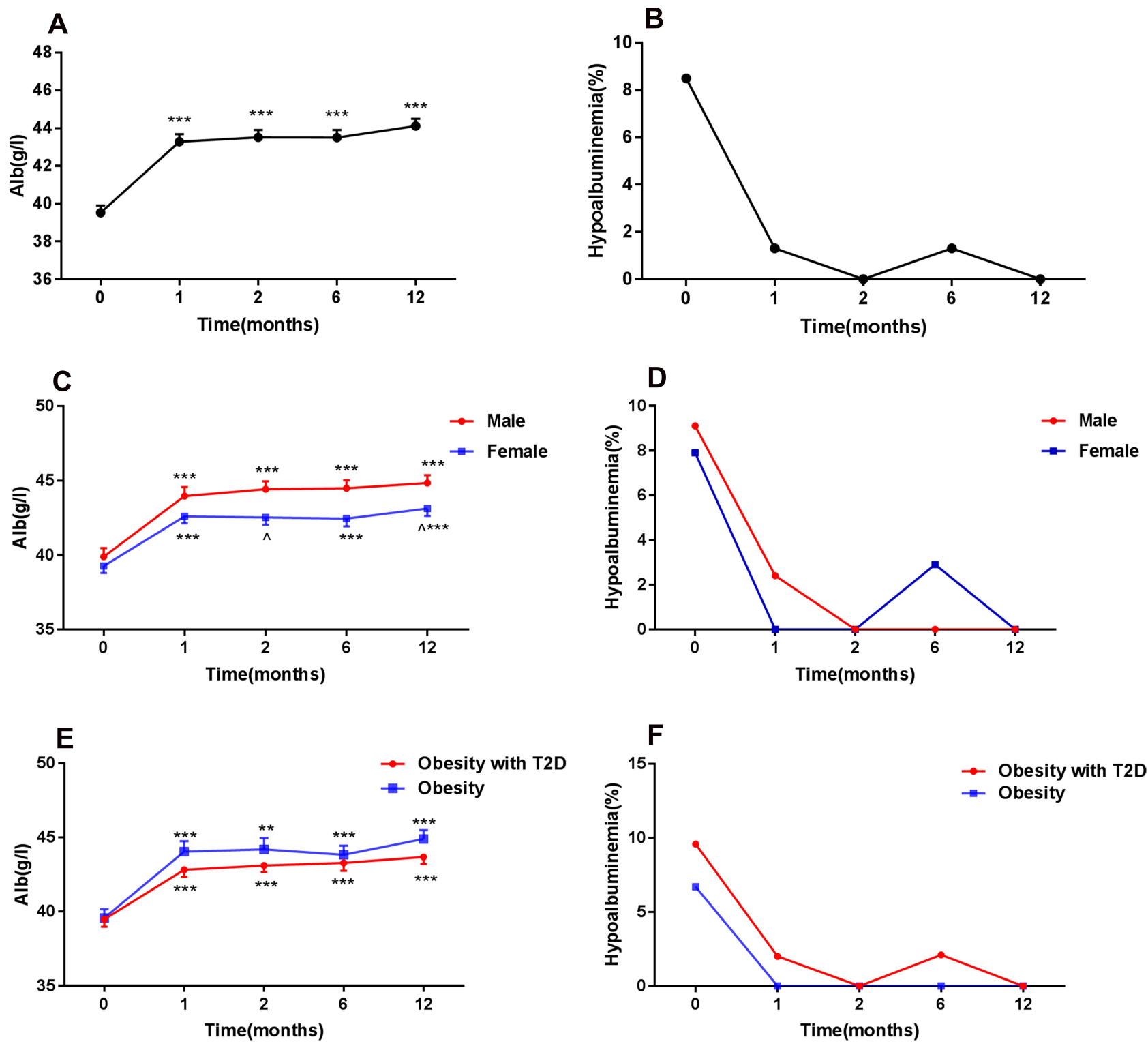

Figure I Changes in serum albumin levels $(\mathbf{A}, \mathbf{C}$ and $\mathbf{E})$ and hypoalbuminemia prevalence $(\mathbf{B}, \mathbf{D}$ and $\mathbf{F})$ in patients after surgery. Data are presented as the mean \pm SEM. $*^{*} \mathrm{p}<0.01, * * * p<0.001$ compared with baseline. ${ }^{\mathrm{p}}<0.05$ compared with males at each time point. Hypoalbuminemia: albumin $<35 \mathrm{~g} / \mathrm{L}$. 
women $(43.2 \pm 2.8 \mathrm{~g} / \mathrm{L}, P=0.023)$ at the 12-month follow-up (Figure 1C).

\section{Anaemia}

The Anaemia Rate Was Higher in the Female Group Than in the Male Group at 12 Months After LSG

No significant differences were found in the $\mathrm{Hb}$ levels at 1-12-month post-surgery compared with the baseline levels in all groups (Tables 1-3, Figure 2A, C and E). For each time point, the $\mathrm{Hb}$ levels in the male group were significantly higher than those in the female group (Table 2 , Figure $2 \mathrm{C}$ ). Anaemia was present in $7.3 \%$ of all patients before surgery, and its prevalence increased to $11.0 \%$ at 12 months post-operation ( $P=0.109)$ (Figure $2 \mathrm{~B}$ ). The percentage of anaemia in each subgroup showed no differences at the 12-month visit (Figure 2D and F). Interestingly, at the last follow-up visit, anaemia was present in 9 patients $(5$, mild; 4, moderate), 1 (2.3\%) male patient and $8(21.1 \%)$ female patients, which indicates that the anaemia rate in females is much higher than that in males $(P=0.036)$. One male and 3 female patients had normocytic anaemia, while the other 5 female had microcytic hypochromic anaemia. Two patients without T2D and 2 with T2D were diagnosed with normocytic anaemia, and the other 5 with T2D patients had microcytic hypochromic anaemia.

\section{No Significant Differences Were Found in the Vitamin BI2 Deficiency Rate Throughout the Study, but the Patients in the Female Group and the Patients without T2D Had a Higher Prevalence of Folate Deficiency After the Surgery}

We used each patient's last follow-up visit to evaluate the use of multivitamin complex supplements. Forty-eight (58.5\%) patients used a multivitamin complex at least occasionally for the prevention and treatment of nutritional deficiencies. Thirty-four (41.4\%) patients were still using their multivitamin supplements at the last follow-up visit. Interestingly, vitamin B12 levels increased at 1 month $(P<0.001)$ after surgery and then decreased to baseline levels at the following visits (Tables 1-3, Figure 3A, $\mathrm{C}$ and $\mathrm{E})$. Nevertheless, there were no significant differences in the prevalence of vitamin B12 deficiency at 12 months after surgery ( $0 \%$ vs $3.8 \%, P=1.0)$ (Figure $3 \mathrm{~B})$. In each subgroup, the percentage of the vitamin B12 deficiency showed no differences at the 12-month visit (Figure $3 \mathrm{D}$ and $\mathrm{F}$ ). Furthermore, females had greater vitamin B12 levels $(524.7 \pm 310.8 \mathrm{pg} / \mathrm{mL})$ at baseline than males $(391.3$ $\pm 111.4 \mathrm{pg} / \mathrm{mL}, P=0.029$ ) (Figure 3C).
No significant changes in folate levels were found postoperation (Tables 1-3, Figure 3G, I and K). Although no significant increase was observed in the folate deficiency rate in all patients $(21.4 \%$ vs $28.9 \%, P>0.05)$ (Figure $3 \mathrm{H}$ ), there were significant increases in folate deficiency in the patients of the female group and the obese without T2D group at 12 months following LSG $(3.7 \%$ vs $20 \%$, $P=0.031 ; 27.3 \%$ vs $47.1 \%, P=0.031$ ) (Figure $3 \mathrm{~J}$ and L).

Ferritin Levels Decreased Significantly After Surgery, and Iron Deficiency at Post-Surgery Increased Among All Patients and in the Subgroup of Female and Obese Patients with T2D

Among all patients, the serum iron levels showed no significant difference at 12 months post-operation (Table 1, Figure 4A). In contrast, the ferritin levels increased significantly at 1 month after surgery and then decreased at the following visits (Tables 1-3, Figure 4B). A significant increase was noted in iron deficiency (defined as ferritin levels $<30 \mathrm{ng} / \mathrm{mL}$ ) across our study ( $2.6 \%$ vs $17.9 \%, P=0.031$ ) (Figure $4 \mathrm{C}$ ). The serum iron and ferritin levels were significantly greater in the male group than in the female group at every visit (Table 2, Figure 4D and E), and the prevalence of iron deficiency was significantly higher in females than in males at 12 months following surgery ( $43.8 \%$ vs $0 \%, \mathrm{P}<0.001)$ (Figure $4 \mathrm{~F})$. In patients with or without $\mathrm{T} 2 \mathrm{D}$, the serum iron and ferritin levels of the two groups were comparable at baseline, but only in patients with T2D, we found a significant decrease in the ferritin level at 12 months post-surgery (Figure 4G and $\mathrm{H}$ ). In addition, a higher rate of iron deficiency was also noted in only patients with obesity and T2D $(27.3 \%$ vs $0 \%, P=0.031)$ (Figure $4 \mathrm{I})$.

\section{Discussion}

After short-term to long-term follow-up studies confirmed the metabolic benefits of bariatric surgery, investigators have paid more attention to the nutritional status of patients with obesity following surgery. Patients who have undergone bariatric surgery are prone to develop nutritional deficiencies due to a restriction in caloric intake and alterations in the gastrointestinal tract. ${ }^{19}$ Hypoalbuminemia, anaemia and related nutrient deficiencies may potentially occur after bariatric surgery. ${ }^{20}$ To our knowledge, this is the first study assessing nutritional status following LSG in Chinese patients.

In our study, during the 12-month visits, we found significant increases in the serum albumin levels and 


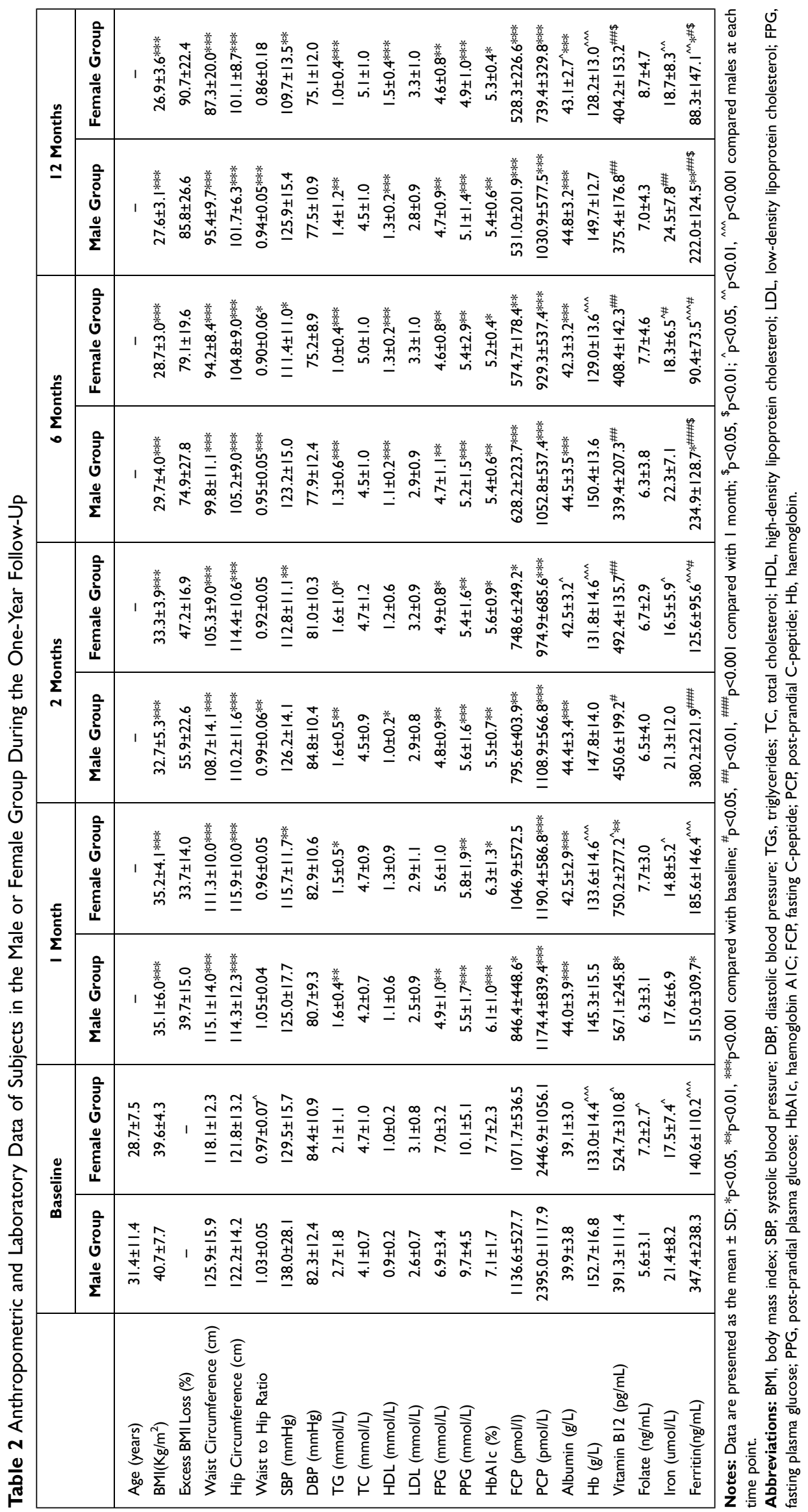




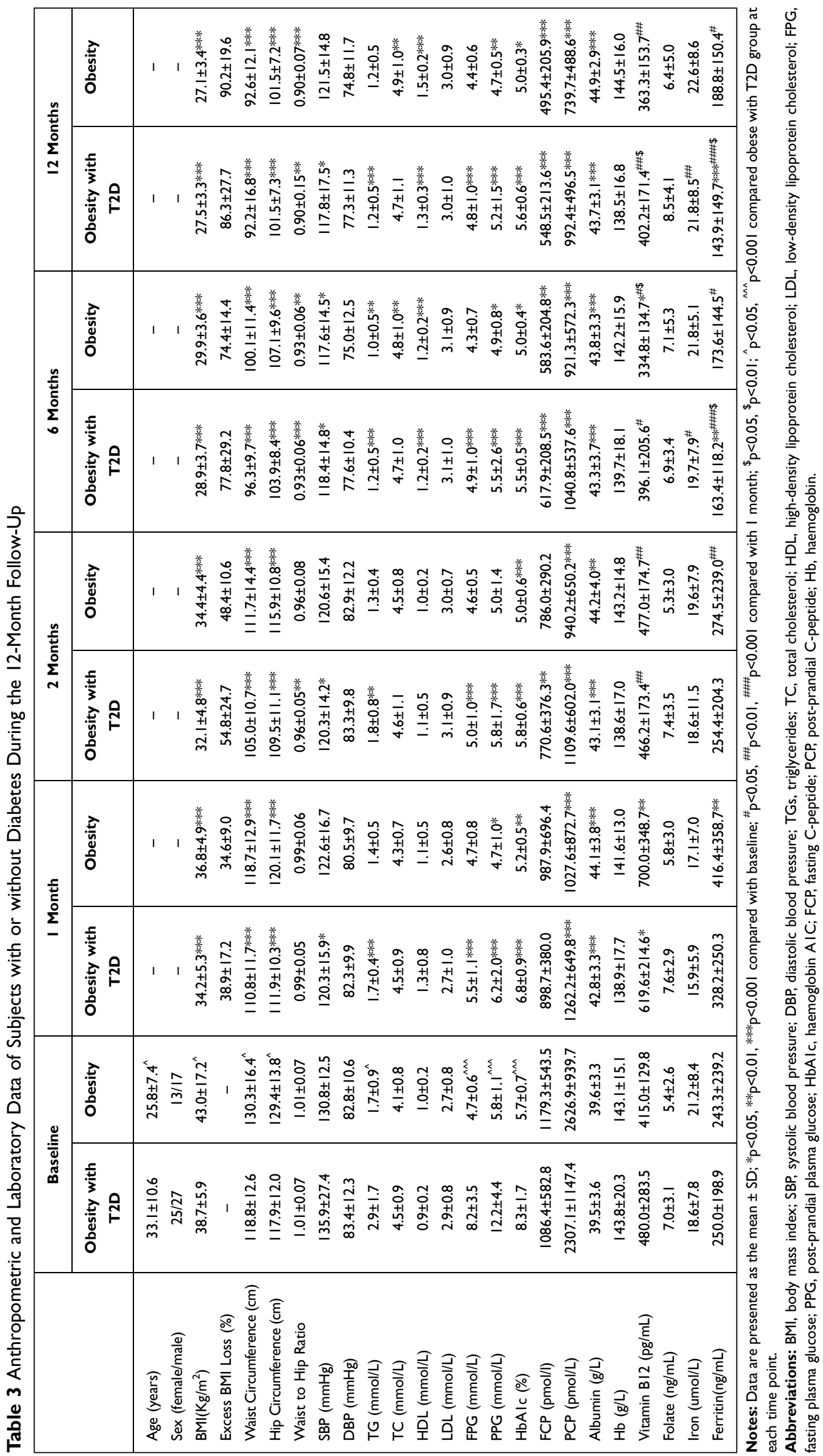



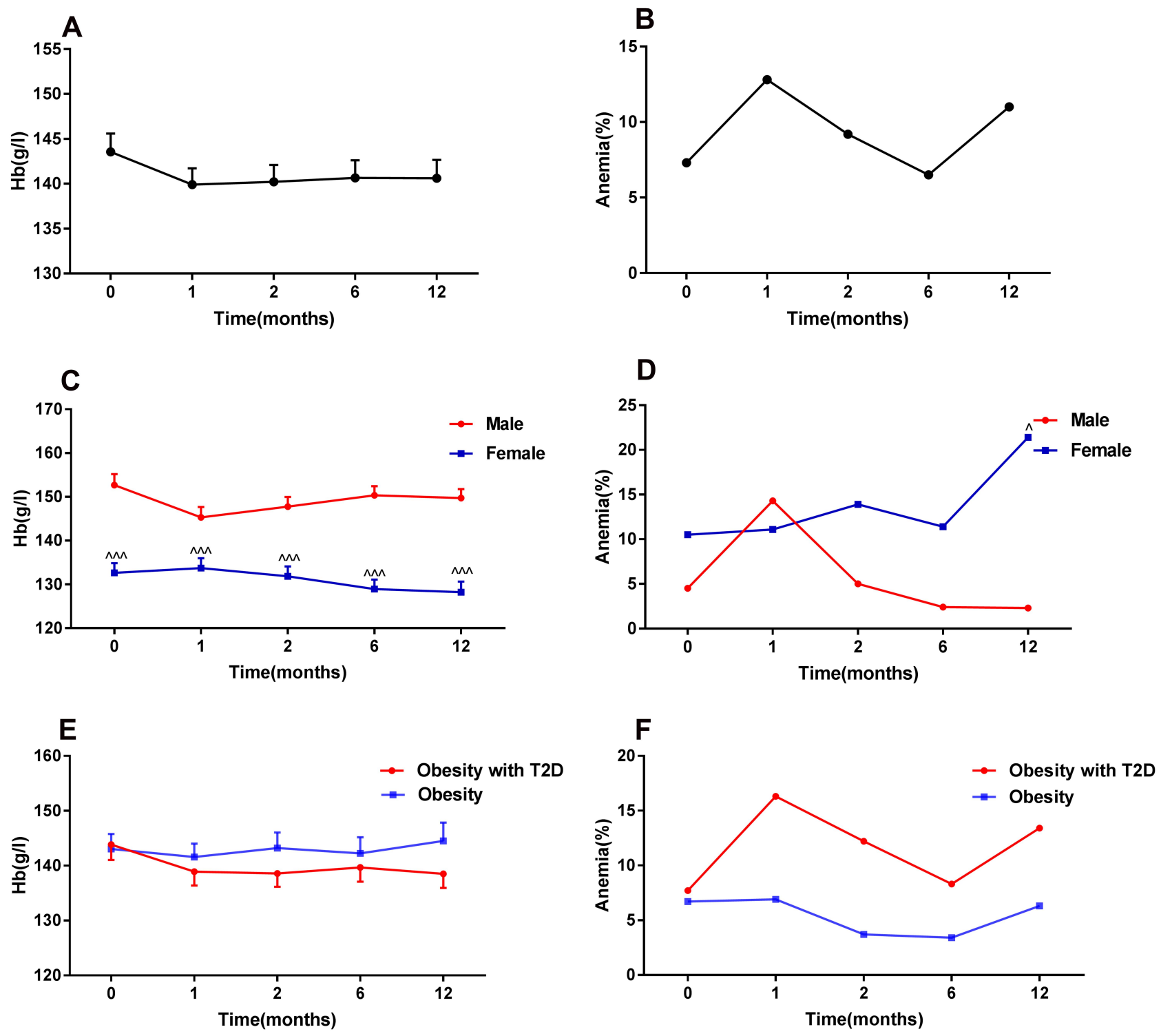

Figure 2 Alterations in the levels of haemoglobin $(\mathbf{A}, \mathbf{C}$ and $\mathbf{E})$ and prevalence of anaemia $(\mathbf{B}, \mathbf{D}$ and $\mathbf{F})$ in patients after surgery. Data are presented as the mean \pm SEM. ${ }^{\wedge} \mathrm{p}<0.05$ compared with males at each time point. ${ }^{\wedge \wedge} \mathrm{p}<0.001$ compared with males at each time point. Anaemia: $\mathrm{Hb}<130 \mathrm{~g} / \mathrm{L}$ in men or $<120 \mathrm{~g} / \mathrm{L}$ in women.

a decrease in the prevalence of albumin deficiency after LSG ( $P=0.063$ ) (Figure 1). Previously, studies showed an increase in the prevalence of hypoalbuminemia or significantly decreased prealbumin concentration after LSG in Canada or in France. ${ }^{12,21}$ Additionally, in Kuwait and Turkey, no significant changes in the albumin level at 1-5 years after LSG were found compared to the baseline values. $^{22,23}$ The discrepancy may be due to the different protein supplements and the different patient populations studied. Adequate protein intake after bariatric surgery is important to enhance weight loss and improve body composition. $^{24}$ Thus, the regular monitoring of serum albumin is strongly recommended to prevent the development of protein malnutrition. Although we found that albumin levels were increased in our patients, which guaranteed the safety of LSG in the Chinese population, long-term results still need to be investigated.

The incidence of anaemia following bariatric surgery has been reported to vary widely across studies because of the complexity of influencing factors, such as different populations, operation types, and supplement protocols. In the current study, no significant changes were noted in the incidence of anaemia following LSG (Figure 2). Two other studies also found that anaemia did not differ significantly at 4 years in Israel $^{25}$ and 1 year in the Netherlands $^{26}$ after LSG. There was evidence 

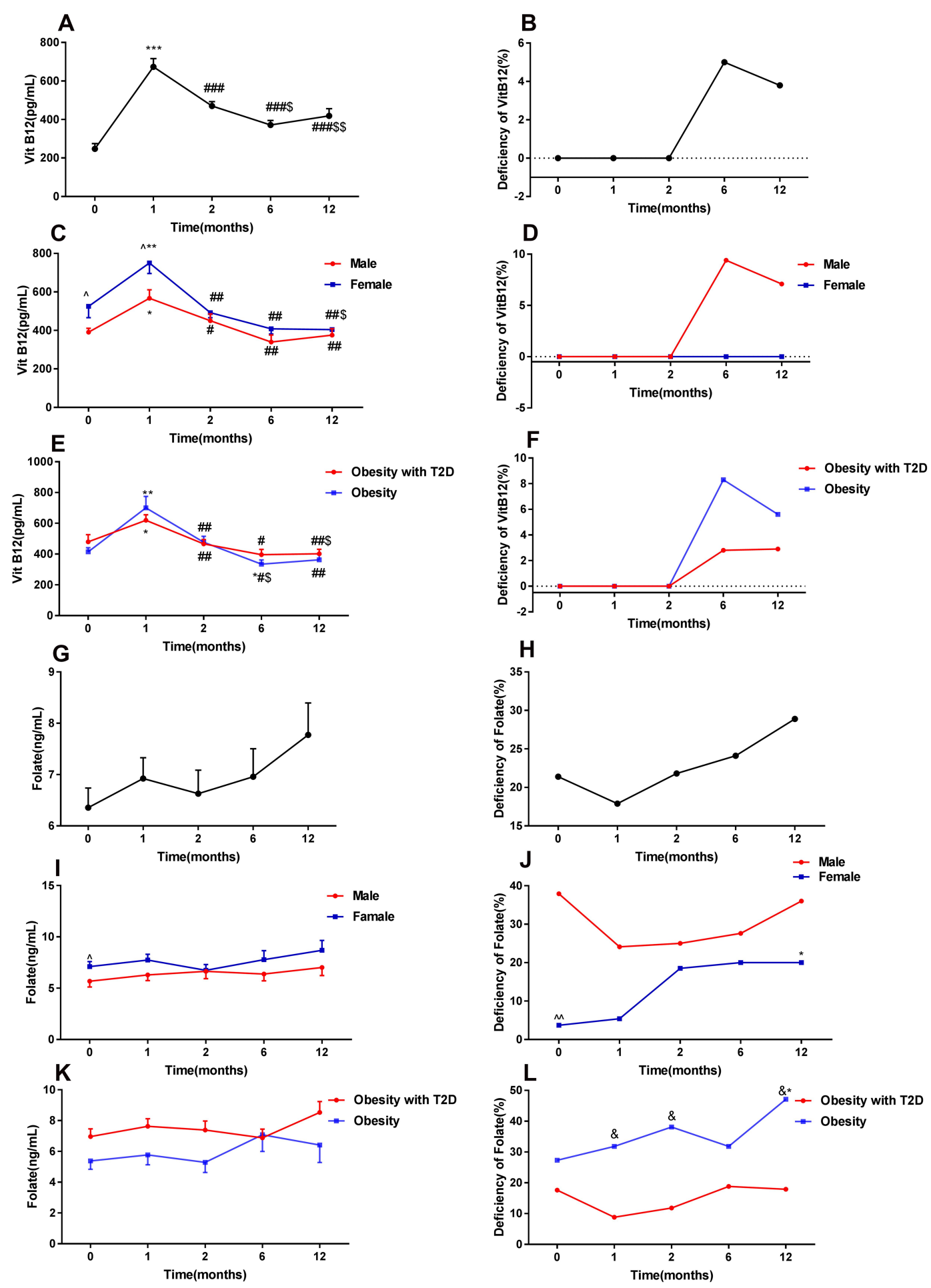

Figure 3 Changes in serum vitamin $B / 2(\mathbf{A}, \mathbf{C}$ and $\mathbf{E})$, folate $(\mathbf{G}, \mathbf{I}$ and $\mathbf{K})$ levels, and prevalence of vitamin $\mathbf{B} \mid 2(\mathbf{B}, \mathbf{D}$ and $\mathbf{F})$, folate $(\mathbf{H}, \mathbf{J}$ and $\mathbf{L})$ deficiency in patients after

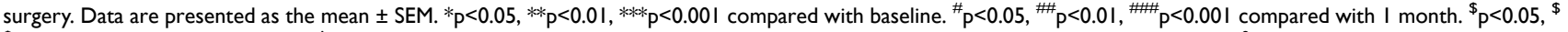
$\$_{p}<0.01$ compared with 2 months. ${ }^{\wedge}<0.05$ compared with males at each time point. ${ }^{\wedge} p<0.01$ compared with males at each time point. ${ }^{*} p<0.05$ compared with obese with T2D at each time point. Vitamin B/2 deficiency: vitamin B/2 $<203 \mathrm{pg} / \mathrm{mL}$; folate deficiency: folate $<4 \mathrm{ng} / \mathrm{mL}$.

demonstrating significantly higher rates of anaemia at 12-20 months post-operation compared to pre-operation rates $(42.9 \%$ vs $16.7 \%)$ in Israel after LRYGB. ${ }^{11}$ Actually, LSG surgery patients in Israel and Netherlands studies were instructed to resume vitamin and mineral supplements in cases of nutritional deficiencies, ${ }^{25,26}$ as well as our study, except for the study of LRYGB surgery in Israel. ${ }^{11}$ Furthermore, compared with LSG, patients after 

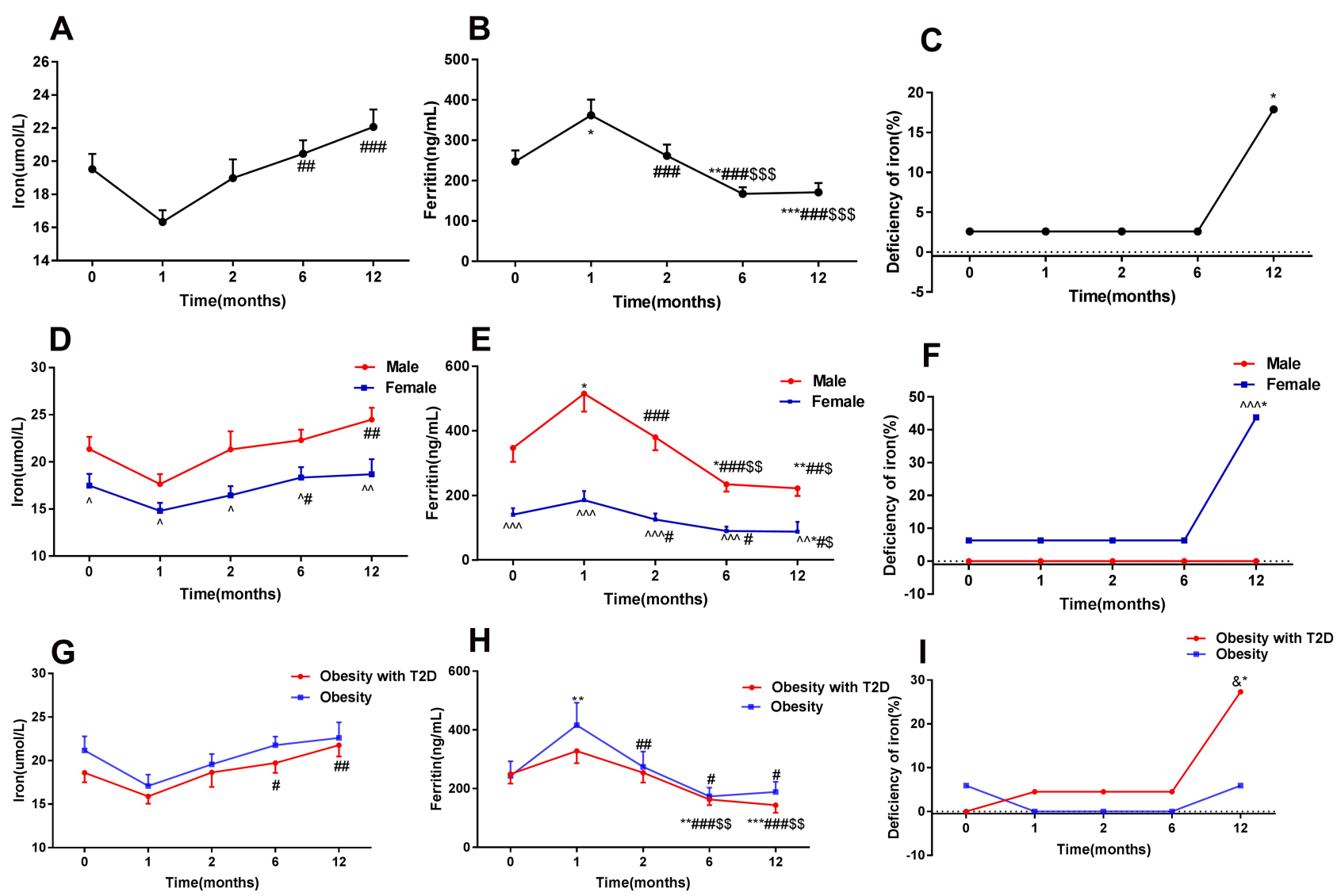

Figure 4 Alterations in the levels of serum iron (A, $\mathbf{D}$ and $\mathbf{G})$, ferritin $(\mathbf{B}, \mathbf{E}$ and $\mathbf{H})$, and prevalence of iron deficiency $(\mathbf{C}, \mathbf{F}$ and $\mathbf{I})$ in patients after surgery. Data are presented as the mean \pm SEM. ${ }^{*} p<0.05,{ }^{*}{ }^{*}<<0.01,{ }^{* * *} \mathrm{p}<0.001$ compared with baseline. ${ }^{\#} \mathrm{p}<0.05,{ }^{\#} \mathrm{p}<0.01$, ${ }^{\#} \mathrm{p}<0.001$ compared with I month. ${ }^{\$} \mathrm{p}<0.05,{ }^{\$} \mathrm{p}<0.0 \mathrm{I},{ }^{\$ \$}$ ${ }_{\mathrm{P}}^{\$}<0.00$ I compared with 2 months. ${ }^{\wedge}<0.05,{ }^{\wedge \wedge} \mathrm{p}<0.01,{ }^{\wedge \wedge} \mathrm{p}<0.001$ compared with males at each time point. ${ }^{\&} \mathrm{p}<0.05$ compared with obese with $\mathrm{T} 2 \mathrm{D}$ at each time point. Iron deficiency was defined as a ferritin level $<30 \mathrm{ng} / \mathrm{mL}$.

LRYGB had a higher prevalence of anaemia, even after adjusting for age and gender. ${ }^{27}$ We also noted that fertile female patients had a higher anaemia prevalence than male patients, and $62.5 \%$ had microcytic hypochromic anaemia, which is consistent with the results that females had lower ferritin and iron levels than males.

Vitamin B12 and folate are both required to sustain normal haematopoiesis. A deficiency of vitamin B12 and/ or folate will cause megaloblastic anaemia. The main reported reason for the vitamin B12 deficiency after bariatric surgery is the reduction in intrinsic factors that are necessary for the absorption of vitamin $\mathrm{B} 12 .{ }^{28}$ The resection of the gastric fundus during LSG leads to a decreased production of intrinsic factors and, as a result, impaired vitamin B12 absorption. ${ }^{29}$ Interestingly, we found that vitamin B12 levels increased at 1 month $(\mathrm{p}<0.001)$ after LSG and then returned to baseline levels at the following visits in the Chinese population (Figure 3). Additionally, a significant decrease in vitamin B12 deficiency at five years after LSG in Canada was demonstrated, ${ }^{12}$ in which the subjects were instructed to take $20-40 \mu \mathrm{g}$ vitamin B12 daily. ${ }^{12}$ Therefore, in our study, the improvement in vitamin B12 levels in the first postoperative month might be the result of the patients' high adherence to vitamin supplements. Another study reported decreased vitamin B12 levels at one year after LSG in Israel. ${ }^{30}$ This difference between our study and a previous study ${ }^{30}$ may be due to vitamin B12 deficiency being absent in our study at baseline, compared to $13.0 \%$ patients in the study mentioned above, ${ }^{30}$ because the deficiencies of vitamin B12 before surgery were predictors for deficiencies after surgery. ${ }^{30}$ The primary reason for folate deficiency is decreased dietary intake, as folate absorption can occur along the entire part of the small intestine. A significant increase was found in the rate of folate deficiency in the patients among the female group and the obese without T2D group throughout our study (Figure 3), while others demonstrated an improvement in folate deficiencies after LRYGB in America or in Denmark. ${ }^{31,32}$ All patients in those studies ${ }^{31,32}$ were supplemented orally with 1 daily 
multivitamin containing folate $(400 \mu \mathrm{g})$, but only $58.5 \%$ patients in our study used a multivitamin complex $(400 \mu \mathrm{g}$ folate/day), which could be the reason for the different results between our study and the previous studies. ${ }^{31,32}$

The serum iron level is an indicator of the iron content, while the ferritin level is an indicator of iron reserves. The low levels of ferritin and iron indicate iron deficiency, which is another important reason for anaemia. Intake restriction and malabsorption might be the main reasons for iron deficiency after bariatric surgery. In addition to assessing the total body iron stores, ferritin is also an acute phase reactant that can be synthesized by the stimulation of multiple cytokines. ${ }^{18}$ Recently, obesity has been proven to be under a status of low-grade, chronic inflammation, which contributes to insulin resistance and $\mathrm{T} 2 \mathrm{D} .{ }^{33}$ Bariatric surgery alleviates the inflammatory status in the circulation, adipose tissue, gut, and even the kidneys of subjects with obesity. ${ }^{34}$ Therefore, the decline in serum ferritin after LSG represents not only a reduction in body iron stores but also an improvement in inflammation. A significant decrease in inflammatory markers (such as interleukin-6 (IL-6) and C-reactive protein) after bariatric surgery was reported, ${ }^{34}$ along with decreased serum transferrin receptor levels. ${ }^{35}$ Furthermore, IL-6 upregulates hepcidin, a peptide that decreases the iron absorption and releases iron from stores. ${ }^{36}$ Thus, the improvement in serum iron found in our study might imply an improvement in iron bioavailability through decreased inflammation. The significant decrease in the serum iron level at 1 month after surgery could be ascribed to the increased inflammation relatively soon after surgery (Figure 4). Furthermore, the serum iron and ferritin levels were significantly greater in the male group than in the fertile female group at every visit (Figure 4). This difference can be attributed to the ongoing menstrual blood loss that only occurs in females. ${ }^{37} \mathrm{We}$ also found a decrease in the ferritin levels and a significant increase in iron deficiency at 6-12 months post-surgery in patients with T2D but not in patients without T2D (Figure 4). A recent study indicated that chronic hyperglycaemia in rats was associated with increased inflammatory responses and oxygen-free radicals ${ }^{38}$ thus, the improvement in glucose metabolism may contribute to decreased inflammation after bariatric surgery, which may explain the difference in the ferritin levels between the T2D group and the nonT2D group after surgery.

In conclusion, our study evaluates the nutritional status following LSG in Chinese patients for the first time. During the 12-month follow-up, our study suggests that LSG is an effective and comparably safe procedure for Chinese patients considering nutritional deficiencies post-surgery.

\section{Conclusions}

In summary, the current data demonstrate that LSG is effective in treating obesity and obesity-associated diseases. Furthermore, based on 12-month visits, we found that the serum albumin levels increased and the ferritin levels decreased significantly after surgery. Although no difference was found in the anaemia rate among all patients, the anaemia rate was higher in the female group than in the male group 12 months after surgery. No difference was noted in the vitamin B12 deficiency. Even though no significant changes were found in the folate and iron levels throughout the study, the deficiency rates were increased in patients of different subgroups: 1) the folate deficiency was increased in the female group and the obese without T2D group, and 2) the iron deficiency was increased in the female group and the obese with T2D group. Thus, these findings reveal that LSG is an effective and comparable safe procedure for Chinese patients, but long-term follow-up and nutritional supplements are also important.

\section{Ethics Approval and Informed Consent}

This study was approved by the ethics committee of the Second Xiangya Hospital of Central South University, and informed consent of the study protocol was provided by all participants.

\section{Funding}

This study was supported by the National Key R\&D Program of China (2016YFC1305000, 2016YFC1 305001), the National Natural Science Foundation of China (82070807, 91749118, 81770775, and 81730022), the Science and Technology Major Project of Hunan Province (2017SK1020), the Planned Science and Technology Project of Hunan Province (2017RS3015), and National key research and development program (2019YFA0801903 and 2018YFC2000100).

\section{Disclosure}

All of the authors disclose no conflicts of interest for this work. 


\section{References}

1. Arnold M, Pandeya N, Byrnes G, et al. Global burden of cancer attributable to high body-mass index in 2012: a population-based study. Lancet Oncol. 2015;16(1):36-46. doi:10.1016/S1470-2045(14)71123-4

2. NCD-RisC NRFC. Trends in adult body-mass index in 200 countries from 1975 to 2014: a pooled analysis of 1698 population-based measurement studies with 19.2 million participants. Lancet. 2016;387(10026):1377-1396. doi:10.1016/S0140-6736(16)30054-X

3. Lu Y, Hajifathalian K, Ezzati M, Woodward M, Rimm EB, Danaei G. Metabolic mediators of the effects of body-mass index, overweight, and obesity on coronary heart disease and stroke: a pooled analysis of 97 prospective cohorts with 1.8 million participants. Lancet. 2014;383(9921):970-983.

4. Wang L, Gao P, Zhang M, et al. Prevalence and ethnic pattern of diabetes and prediabetes in China in 2013. JAMA. 2017;317 (24):2515-2523. doi:10.1001/jama.2017.7596

5. Adams TD, Davidson LE, Litwin SE, et al. Weight and metabolic outcomes 12 years after gastric bypass. $N$ Engl J Med. 2017;377 (12):1143-1155. doi:10.1056/NEJMoa1700459

6. Peterli R, Wolnerhanssen BK, Peters T, et al. Effect of laparoscopic sleeve gastrectomy vs laparoscopic Roux-en-Y Gastric bypass on weight loss in patients with morbid obesity: the SM-BOSS randomized clinical trial. JAMA. 2018;319(3):255-265. doi:10.1001/ jama.2017.20897

7. Salminen P, Helmio M, Ovaska J, et al. Effect of laparoscopic sleeve gastrectomy vs laparoscopic Roux-en-Y gastric bypass on weight loss at 5 years among patients with morbid obesity: the SLEEVEPASS randomized clinical trial. JAMA. 2018;319(3):241-254. doi:10.1001/ jama.2017.20313

8. Shen X, Zhang X, Bi J, Yin K. Long-term complications requiring reoperations after laparoscopic adjustable gastric banding: a systematic review. Surg Obes Relat Dis. 2015;11(4):956-964. doi:10.1016/j.soard.2014.11.011

9. Mingrone G, Panunzi S, De Gaetano A, et al. Bariatric-metabolic surgery versus conventional medical treatment in obese patients with type 2 diabetes: 5 year follow-up of an open-label, single-centre, randomised controlled trial. Lancet. 2015;386(9997):964-973. doi:10.1016/S0140-6736(15)00075-6

10. Risstad H, Sovik TT, Engstrom M, et al. Five-year outcomes after laparoscopic gastric bypass and laparoscopic duodenal switch in patients with body mass index of 50 to 60: a randomized clinical trial. JAMA Surg. 2015;150(4):352-361. doi:10.1001/jamasurg.2014.3579

11. Kessler Y, Adelson D, Mardy-Tilbor L, et al. Nutritional status following one anastomosis gastric bypass. Clin Nutr. 2020;39 (2):599-605. doi:10.1016/j.clnu.2019.03.008

12. Caron M, Hould FS, Lescelleur O, et al. Long-term nutritional impact of sleeve gastrectomy. Surg Obes Relat Dis. 2017;13(10):1664-1673. doi:10.1016/j.soard.2017.07.019

13. Yu H, Du R, Zhang N, et al. Iron-deficiency anemia after laparoscopic Roux-en-Y gastric bypass in chinese obese patients with type 2 diabetes: a 2-year follow-up study. Obes Surg. 2016;26 (11):2705-2711. doi:10.1007/s11695-016-2161-9

14. Rubino F, Nathan DM, Eckel RH, et al. Metabolic surgery in the treatment algorithm for type 2 diabetes: a joint statement by international diabetes organizations. Diabetes Care. 2016;39(6):861-877. doi:10.2337/dc16-0236

15. Alberti KG, Zimmet PZ. Definition, diagnosis and classification of diabetes mellitus and its complications. Part 1: diagnosis and classification of diabetes mellitus provisional report of a WHO consultation. Diabet Med. 1998;15(7):539-553. doi:10.1002/(SICI) 1096-9136(199807)15:7<539::AID-DIA668>3.0.CO;2-S

16. World Health Organization. Haemoglobin concentrations for the diagnosis of anaemia and assessment of severity. Geneva: World Health Organization; 2011. Available from: https://www.who.int/ vmnis/indicators/haemoglobin/en/. Accessed march 17, 2020.
17. de Benoist B. Conclusions of a WHO Technical Consultation on folate and vitamin B12 deficiencies. Food Nutr Bull. 2008;29(2 Suppl):S238-44. doi:10.1177/15648265080292S129

18. Weiss G, Goodnough LT. Anemia of chronic disease. $N$ Engl J Med. 2005;352(10):1011-1023. doi:10.1056/NEJMra041809

19. Stroh C, Manger T, Benedix F. Metabolic surgery and nutritional deficiencies. Minerva Chir. 2017;72(5):432-441. doi:10.23736/S00 26-4733.17.07408-9

20. Ziegler O, Sirveaux MA, Brunaud L, Reibel N, Quilliot D. Medical follow up after bariatric surgery: nutritional and drug issues. General recommendations for the prevention and treatment of nutritional deficiencies. Diabetes Metab. 2009;35(6 Pt 2):544-557. doi:10.10 16/S1262-3636(09)73464-0

21. Verger EO, Aron-Wisnewsky J, Dao MC, et al. Micronutrient and protein deficiencies after gastric bypass and sleeve gastrectomy: a 1-year follow-up. Obes Surg. 2016;26(4):785-796. doi:10.1007/ s11695-015-1803-7

22. Al-Mutawa A, Al-Sabah S, Anderson AK, Al-Mutawa M. Evaluation of nutritional status post laparoscopic sleeve gastrectomy-5-year outcomes. Obes Surg. 2018;28(6):1473-1483. doi:10.1007/s11695017-3041-7

23. Mihmanli M, Isil RG, Isil CT, et al. Effects of laparoscopic sleeve gastrectomy on parathyroid hormone, vitamin D, calcium, phosphorus, and albumin levels. Obes Surg. 2017;27(12):3149-3155. doi: $10.1007 / \mathrm{s} 11695-017-2747-\mathrm{x}$

24. Faria SL, Faria OP, Buffington C, de Almeida CM, Ito MK. Dietary protein intake and bariatric surgery patients: a review. Obes Surg. 2011;21(11):1798-1805. doi:10.1007/s11695-011-0441-y

25. Ben-Porat T, Elazary R, Goldenshluger A, Sherf DS, Mintz Y, Weiss R. Nutritional deficiencies four years after laparoscopic sleeve gastrectomy-are supplements required for a lifetime? Surg Obes Relat Dis. 2017;13(7):1138-1144. doi:10.1016/j.soard.2017.02.021

26. van Rutte PW, Aarts EO, Smulders JF, Nienhuijs SW. Nutrient deficiencies before and after sleeve gastrectomy. Obes Surg. 2014;24(10):1639-1646. doi:10.1007/s11695-014-1225-y

27. Bailly L, Schiavo L, Sebastianelli L, Fabre R, Pradier C, Iannelli A. Anemia and bariatric surgery: results of a National French survey on administrative data of 306,298 consecutive patients between 2008 and 2016. Obes Surg. 2018;28(8):2313-2320. doi:10.1007/s11695018-3143-x

28. Sala P, Belarmino G, Torrinhas RS, et al. Gastrointestinal transcriptomic response of metabolic vitamin B12 pathways in Roux-en-Y gastric bypass. Clin Transl Gastroenterol. 2017;8(1):e212. doi:10. $1038 /$ ctg. 2016.67

29. Snyder-Marlow G, Taylor D, Lenhard MJ. Nutrition care for patients undergoing laparoscopic sleeve gastrectomy for weight loss. $J \mathrm{Am}$ Diet Assoc. 2010;110(4):600-607. doi:10.1016/j.jada.2009.12.022

30. Ben-Porat T, Elazary R, Yuval JB, Wieder A, Khalaileh A, Weiss R. Nutritional deficiencies after sleeve gastrectomy: can they be predicted preoperatively? Surg Obes Relat Dis. 2015;11(5):1029-1036. doi:10.1016/j.soard.2015.02.018

31. von Drygalski A, Andris DA, Nuttleman PR, Jackson S, Klein J, Wallace JR. Anemia after bariatric surgery cannot be explained by iron deficiency alone: results of a large cohort study. Surg Obes Relat Dis. 2011;7(2):151-156. doi:10.1016/j.soard.2010.04.008

32. Kornerup LS, Hvas CL, Abild CB, Richelsen B, Nexo E. Early changes in vitamin B12 uptake and biomarker status following Roux-en-Y gastric bypass and sleeve gastrectomy. Clin Nutr. 2019;38(2):906-911. doi:10.1016/j.clnu.2018.02.007

33. Asghar A, Sheikh N. Role of immune cells in obesity induced low grade inflammation and insulin resistance. Cell Immunol. 2017;315:18-26. doi:10.1016/j.cellimm.2017.03.001

34. Zhang C, Zhang J, Liu Z, Zhou Z. More than an anti-diabetic bariatric surgery, metabolic surgery alleviates systemic and local inflammation in obesity. Obes Surg. 2018;28(11):3658-3668. doi:10.1007/s11695-018-3400-z 
35. Tussing-Humphreys LM, Nemeth E, Fantuzzi G, et al. Decreased serum hepcidin and improved functional iron status 6 months after restrictive bariatric surgery. Obesity. 2010;18(10):2010-2016. doi:10. 1038/oby. 2009.490

36. Ganz T. Molecular control of iron transport. J Am Soc Nephrol. 2007;18(2):394-400. doi:10.1681/ASN.2006070802

37. Ross EM. Evaluation and treatment of iron deficiency in adults. Nutr Clin Care. 2002;5(5):220-224. doi:10.1046/j.1523-5408.2002.05503.x
38. Park KS, Kim JB, Keung M, et al. Chronic hyperglycemia before spinal cord injury increases inflammatory reaction and astrogliosis after injury: human and rat studies. J Neurotrauma. 2020;37 (9):1165-1181. doi:10.1089/neu.2019.6724

Diabetes, Metabolic Syndrome and Obesity: Targets and Therapy

\section{Publish your work in this journal}

Diabetes, Metabolic Syndrome and Obesity: Targets and Therapy is an international, peer-reviewed open-access journal committed to the rapid publication of the latest laboratory and clinical findings in the fields of diabetes, metabolic syndrome and obesity research. Original research, review, case reports, hypothesis formation, expert opinion and commentaries are all considered for publication. The manuscript management system is completely online and includes a very quick and fair peer-review system, which is all easy to use. Visit http://www.dovepress.com/testimonials.php to read real quotes from published authors. 